\title{
On the oscillation of Hadamard fractional differential equations
}

\author{
Bahaaeldin Abdalla ${ }^{1}$ and Thabet Abdeljawad ${ }^{1 *}$ (D)
}

*Correspondence: tabdeljawad@psu.edu.sa

${ }^{1}$ Department of Mathematics and General Sciences, Prince Sultan

University, Riyadh, Saudi Arabia

\section{Springer}

\begin{abstract}
Hadamard fractional derivatives are nonlocal fractional derivatives with singular logarithmic kernel with memory, and hence they are suitable to describe complex systems. In this paper, sufficient conditions are established for the oscillation of solutions fractional differential equations in the frame of left Hadamard fractional derivatives of order $\alpha \in \mathbb{C}, \operatorname{Re}(\alpha) \geq 0$. The results are also obtained for fractional Hadamard derivatives in the Caputo setting. Examples are provided to illustrate the applicability of the main results.
\end{abstract}

MSC: 34A08; 34C10; 26A33

Keywords: Fractional Hadamard integrals; Fractional Hadamard derivatives; Fractional differential equations; Oscillation theory

\section{Introduction}

The oscillation theory for fractional differential and difference equations was studied by some authors (see [1-8]). The study of oscillation and other qualitative properties of fractional dynamical systems such as stability, existence, and uniqueness of solutions is necessary to analyze the systems under consideration [9-13]. The analysis of the memory of certain fractional dynamical systems has gained a high impact in the last few years $[14,15]$. Several definitions of fractional derivatives and fractional integral operators with different kernels exist in the literature. The Hadamard definition, which depends on a logarithmic type kernel, was introduced in [16], and later on some authors contributed to the development of its theory (see [17-19]). In this paper, we study the oscillation of Hadamard fractional differential equation of the form

$$
\left\{\begin{array}{l}
\mathcal{D}_{a}^{\alpha} x(t)+f_{1}(t, x)=r(t)+f_{2}(t, x), \quad t>a \\
\lim _{t \rightarrow a^{+}} \mathcal{D}_{a}^{\alpha-j} x(t)=b_{j} \quad(j=1,2, \ldots, n),
\end{array}\right.
$$

where $n=\lceil\alpha\rceil, \mathcal{D}_{a}^{\alpha}$ is the left-fractional Hadamard derivative of order $\alpha \in \mathbb{C}, \operatorname{Re}(\alpha) \geq 0$ in the Riemann-Liouville setting.

The objective of this paper is to study the oscillation of Hadamard fractional differential equations of the form (1) besides their Caputo setting.

This paper is organized as follows. Section 2 introduces some notations and provides the definitions of Hadamard fractional integral and differential operators together with some basic properties and lemmas that are needed in the proofs of the main theorems.

(c) The Author(s) 2018. This article is distributed under the terms of the Creative Commons Attribution 4.0 International License (http://creativecommons.org/licenses/by/4.0/), which permits unrestricted use, distribution, and reproduction in any medium, provided you give appropriate credit to the original author(s) and the source, provide a link to the Creative Commons license, and indicate if changes were made. 
In Sect. 3, the main theorems are presented. Section 4 is devoted to the results obtained for Hadamard fractional operators in the Caputo setting. Finally, examples are provided in Sect. 5 to explain the effectiveness of the main results.

\section{Notations and preliminary assertions}

We start this section by introducing the definition of the Hadamard type fractional integrals and derivatives (see [20]).

Let $(a, b)$ be a finite or infinite interval of $\mathbb{R}^{+}$starting from $a$.The left-sided Hadamard fractional integral of order $\alpha \in \mathbb{C}, \operatorname{Re}(\alpha)>0$ is defined by

$$
\left(\mathcal{I}_{a}^{\alpha} f\right)(x):=\frac{1}{\Gamma(\alpha)} \int_{a}^{x}\left(\ln \frac{x}{t}\right)^{\alpha-1} \frac{f(t)}{t} d t \quad(a<x<b) .
$$

The right-sided Hadamard fractional integral of order $\alpha \in \mathbb{C}, \operatorname{Re}(\alpha)>0$ is defined by

$$
\left(\mathcal{I}_{b}^{\alpha} f\right)(x):=\frac{1}{\Gamma(\alpha)} \int_{x}^{b}\left(\ln \frac{t}{x}\right)^{\alpha-1} \frac{f(t)}{t} d t \quad(a<x<b) .
$$

The delta derivative is defined by $\delta=x D$, where $D=\frac{d}{d x}$. The left-sided Hadamard fractional derivative of order $\alpha \in \mathbb{C}, \operatorname{Re}(\alpha)>0, n=\lceil\operatorname{Re}(\alpha)\rceil$ is defined by

$$
\begin{aligned}
\left(\mathcal{D}_{a}^{\alpha} y\right)(x) & :=\delta^{n}\left(\mathcal{I}_{a}^{n-\alpha} y\right)(x) \\
& =\left(x \frac{d}{d x}\right)^{n} \frac{1}{\Gamma(n-\alpha)} \int_{a}^{x}\left(\ln \frac{x}{t}\right)^{n-\alpha-1} \frac{y(t)}{t} d t \quad(a<x<b) .
\end{aligned}
$$

The right-sided Hadamard fractional derivative of order $\alpha \in \mathbb{C}, \operatorname{Re}(\alpha)>0, n=\lceil\operatorname{Re}(\alpha)\rceil$ is defined by

$$
\begin{aligned}
\left(\mathcal{D}_{b}^{\alpha} y\right)(x) & :=(-\delta)^{n}\left(\mathcal{I}_{b}^{n-\alpha} y\right)(x) \\
& =\left(-x \frac{d}{d x}\right)^{n} \frac{1}{\Gamma(n-\alpha)} \int_{x}^{b}\left(\ln \frac{t}{x}\right)^{n-\alpha-1} \frac{y(t)}{t} d t \quad(a<x<b) .
\end{aligned}
$$

Likewise Riemann-Liouville and Caputo fractional operators, left and right Hadamard type fractional operators are dual to each other via the $Q$-operator $[21,22](Q f(t)=f(a+$ $b-t)$ ).

Now, we recall the definitions of Caputo type Hadamard fractional derivatives (see [17]).

Definition 2.1 ([17]) The left- and right-sided Hadamard fractional derivatives of Caputo type respectively are defined by

$$
{ }^{C} \mathcal{D}_{a}^{\alpha} y(x)=\frac{1}{\Gamma(n-\alpha)} \int_{a}^{x}\left(\ln \frac{x}{t}\right)^{n-\alpha-1} \frac{\delta^{n} y(t)}{t} d t=\mathcal{I}_{a}^{n-\alpha} \delta^{n} y(x)
$$

and

$$
{ }^{C} \mathcal{D}_{b}^{\alpha} y(x)=\frac{(-1)^{n}}{\Gamma(n-\alpha)} \int_{x}^{b}\left(\ln \frac{t}{x}\right)^{n-\alpha-1} \frac{\delta^{n} y(t)}{t} d t=(-1)^{n} \mathcal{I}_{b}^{n-\alpha} \delta^{n} y(x),
$$

where $\operatorname{Re}(\alpha)>0, n=\lceil\operatorname{Re}(\alpha)\rceil$, and $y(x) \in A C_{\delta}^{n}[a, b]$. 
The following properties are useful in the sequel.

Property $2.1([17,20])$ Let $n=\lceil\alpha\rceil, \operatorname{Re}(\alpha) \geq 0$, and $\operatorname{Re}(\beta)>0$. Then

1. $\mathcal{D}_{a}^{\alpha}\left(\ln \frac{x}{a}\right)^{\beta-1}=\frac{\Gamma(\beta)}{\Gamma(\beta-\alpha)}\left(\ln \frac{x}{a}\right)^{\beta-\alpha-1}$,

2. $\mathcal{D}_{b}^{\alpha}\left(\ln \frac{b}{x}\right)^{\beta-1}=\frac{\Gamma(\beta)}{\Gamma(\beta-\alpha)}\left(\ln \frac{b}{x}\right)^{\beta-\alpha-1}$,

3. $\mathcal{D}_{a}^{\alpha}\left(\ln \frac{x}{a}\right)^{\alpha-n}=0$ and $\mathcal{D}_{b}^{\alpha}\left(\ln \frac{b}{x}\right)^{\alpha-n}=0$,

4. ${ }^{C} \mathcal{D}_{a}^{\alpha}\left(\ln \frac{x}{a}\right)^{\beta-1}=\frac{\Gamma(\beta)}{\Gamma(\beta-\alpha)}\left(\ln \frac{x}{a}\right)^{\beta-\alpha-1}, \operatorname{Re}(\beta)>n$,

5. ${ }^{C} \mathcal{D}_{b}^{\alpha}\left(\ln \frac{b}{x}\right)^{\beta-1}=\frac{\Gamma(\beta)}{\Gamma(\beta-\alpha)}\left(\ln \frac{b}{x}\right)^{\beta-\alpha-1}, \operatorname{Re}(\beta)>n$,

6. ${ }^{C} \mathcal{D}_{a}^{\alpha}\left(\ln \frac{x}{a}\right)^{k}=0$ and ${ }^{C} \mathcal{D}_{b}^{\alpha}\left(\ln \frac{b}{x}\right)^{k}=0$ for $k=0,1, \ldots, n-1$,

7. $\mathcal{D}_{a}^{\alpha} 1=\frac{1}{\Gamma(1-\alpha)}\left(\ln \frac{x}{a}\right)^{-\alpha}$ and ${ }^{C} \mathcal{D}_{a}^{\alpha} 1=0$.

Lemma 2.1 ([23] Young's inequality)

(i) Let $X, Y \geq 0, u>1$, and $\frac{1}{u}+\frac{1}{v}=1$, then $X Y \leq \frac{1}{u} X^{u}+\frac{1}{v} Y^{v}$.

(ii) Let $X \geq 0, Y>0,0<u<1$, and $\frac{1}{u}+\frac{1}{v}=1$, then $X Y \geq \frac{1}{u} X^{u}+\frac{1}{v} Y^{v}$, where equalities hold if and only if $Y=X^{u-1}$.

\section{Oscillation of Hadamard fractional differential equations in the frame of Riemann}

In this section we study the oscillation theory for equation (1).

Lemma 3.1 ([20]) Let $\operatorname{Re}(\alpha)>0, n=-[-\operatorname{Re}(\alpha)], y(x) \in L(a, b)$, and $\left(\mathcal{I}_{a}^{n-\alpha} y\right)(x) \in A C_{\delta}^{n}[a, b]$. Then

$$
\left(\mathcal{I}_{a}^{\alpha} \mathcal{D}_{a}^{\alpha} y\right)(x)=y(x)-\sum_{j=1}^{n} \frac{\left(\delta^{n-j} \mathcal{I}_{a}^{n-\alpha} y\right)(a)}{\Gamma(\alpha-j+1)}\left(\ln \frac{x}{a}\right)^{\alpha-j}
$$

Using Lemma 3.1, the solution representation of (1) can be written as

$$
x(t)=\sum_{j=1}^{n} \frac{\left(\mathcal{D}_{a}^{\alpha-j} x\right)(a)}{\Gamma(\alpha-j+1)}\left(\ln \frac{t}{a}\right)^{\alpha-j}+\mathcal{I}_{a}^{\alpha} F(t, x),
$$

where $F(t, x)=r(t)+f_{2}(t, x)-f_{1}(t, x)$.

A solution of (1) is said to be oscillatory if it has arbitrarily large zeros on $(0, \infty)$; otherwise, it is called nonoscillatory. An equation is said to be oscillatory if all of its solutions are oscillatory.

We prove our results under the following assumptions:

$$
\begin{aligned}
& x f_{i}(t, x)>0 \quad(i=1,2), x \neq 0, t \geq 0, \\
& \left|f_{1}(t, x)\right| \geq p_{1}(t)|x|^{\beta} \quad \text { and } \quad\left|f_{2}(t, x)\right| \leq p_{2}(t)|x|^{\gamma}, \quad x \neq 0, t \geq 0, \\
& \left|f_{1}(t, x)\right| \leq p_{1}(t)|x|^{\beta} \quad \text { and } \quad\left|f_{2}(t, x)\right| \geq p_{2}(t)|x|^{\gamma}, \quad x \neq 0, t \geq 0,
\end{aligned}
$$

where $p_{1}, p_{2} \in C([0, \infty),(0, \infty))$, and $\beta, \gamma$ are positive constants.

Define

$$
\Phi(t)=\Gamma(\alpha) \sum_{j=1}^{n} \frac{\left(\mathcal{D}_{a}^{\alpha-j} x\right)(a)}{\Gamma(\alpha-j+1)}\left(\ln \frac{t}{a}\right)^{\alpha-j}
$$


and

$$
\Psi\left(t, T_{1}\right)=\int_{a}^{T_{1}}\left(\ln \frac{t}{s}\right)^{\alpha-1} \frac{F(s, x(s))}{s} d s
$$

Theorem 3.2 Let $f_{2}=0$ in (1) and condition (10) hold. If

$$
\liminf _{t \rightarrow \infty}(\ln t)^{1-\alpha} \int_{T}^{t}\left(\ln \frac{t}{s}\right)^{\alpha-1} \frac{r(s)}{s} d s=-\infty
$$

and

$$
\limsup _{t \rightarrow \infty}(\ln t)^{1-\alpha} \int_{T}^{t}\left(\ln \frac{t}{s}\right)^{\alpha-1} \frac{r(s)}{s} d s=\infty
$$

for every sufficiently large $T$, then every solution of (1) is oscillatory.

Proof Let $x(t)$ be a nonoscillatory solution of equation (1) with $f_{2}=0$. Suppose that $T_{1}>a$ is large enough so that $x(t)>0$ for $t \geq T_{1}$. Hence, (10) implies that $f_{1}(t, x)>0$ for $t \geq T_{1}$. Using (2), we get from (9)

$$
\begin{aligned}
\Gamma(\alpha) x(t)= & \Gamma(\alpha) \sum_{j=1}^{n} \frac{\left(\mathcal{D}^{\alpha-j} x\right)(a)}{\Gamma(\alpha-j+1)}\left(\ln \frac{t}{a}\right)^{\alpha-j} \\
& +\int_{a}^{T_{1}}\left(\ln \frac{t}{s}\right)^{\alpha-1} \frac{F(s, x(s))}{s} d s \\
& +\int_{T_{1}}^{t}\left(\ln \frac{t}{s}\right)^{\alpha-1} \frac{\left[r(s)-f_{1}(s, x(s))\right]}{s} d s \\
\leq & \Phi(t)+\Psi\left(t, T_{1}\right)+\int_{T_{1}}^{t}\left(\ln \frac{t}{s}\right)^{\alpha-1} \frac{r(s)}{s} d s
\end{aligned}
$$

where $\Phi$ and $\Psi$ are defined in (13) and (14), respectively.

Multiplying (17) by $(\ln t)^{1-\alpha}$, we get

$$
\begin{aligned}
0< & (\ln t)^{1-\alpha} \Gamma(\alpha) x(t) \leq(\ln t)^{1-\alpha} \Phi(t)+(\ln t)^{1-\alpha} \Psi\left(t, T_{1}\right) \\
& +(\ln t)^{1-\alpha} \int_{T_{1}}^{t}\left(\ln \frac{t}{s}\right)^{\alpha-1} \frac{r(s)}{s} d s
\end{aligned}
$$

Take $T_{2}>T_{1}$. We consider two cases as follows.

Case (1): Let $0<\alpha \leq 1$. Then $n=1$ and $\left|(\ln t)^{1-\alpha} \Phi(t)\right|=\left|b_{1}(\ln t)^{1-\alpha}\left(\ln \frac{t}{a}\right)^{\alpha-1}\right|$.

Since $h_{1}(t)=\left(\frac{\ln t-\ln a}{\ln t}\right)^{\alpha-1}$ is decreasing for $a>1$ and $t>T_{2}$, we get

$$
\left|(\ln t)^{1-\alpha} \Phi(t)\right|=\left|b_{1}\left(\frac{\ln t-\ln a}{\ln t}\right)^{\alpha-1}\right| \leq\left|b_{1}\right|\left(\frac{\ln T_{2}-\ln a}{\ln T_{2}}\right)^{\alpha-1}=c_{1}\left(T_{2}\right)
$$

We also have

$$
\left|(\ln t)^{1-\alpha} \Psi\left(t, T_{1}\right)\right|
$$




$$
\begin{aligned}
& =\left|(\ln t)^{1-\alpha} \int_{a}^{T_{1}}\left(\ln \frac{t}{s}\right)^{\alpha-1} \frac{\left[r(s)+f_{2}(s, x(s))-f_{1}(s, x(s))\right]}{s} d s\right| \\
& \leq \int_{a}^{T_{1}}\left(\frac{\ln t-\ln s}{\ln t}\right)^{\alpha-1} \frac{\left|r(s)+f_{2}(s, x(s))-f_{1}(s, x(s))\right|}{s} d s \\
& \leq \int_{a}^{T_{1}}\left(\frac{\ln T_{2}-\ln s}{\ln T_{2}}\right)^{\alpha-1} \frac{\left|r(s)+f_{2}(s, x(s))-f_{1}(s, x(s))\right|}{s} d s \\
& :=c_{2}\left(T_{1}, T_{2}\right) .
\end{aligned}
$$

Then from equation (18) we get

$$
(\ln t)^{1-\alpha} \int_{T_{1}}^{t}\left(\ln \frac{t}{s}\right)^{\alpha-1} \frac{r(s)}{s} d s \geq-\left[c_{1}\left(T_{1}\right)+c_{2}\left(T_{1}, T_{2}\right)\right]
$$

hence

$$
\liminf _{t \rightarrow \infty}(\ln t)^{1-\alpha} \int_{T_{1}}^{t}\left(\ln \frac{t}{s}\right)^{\alpha-1} \frac{r(s)}{s} d s \geq-\left[c_{1}\left(T_{1}\right)+c_{2}\left(T_{1}, T_{2}\right)\right]>-\infty
$$

which contradicts condition (15).

Case (2): Let $\alpha>1$. Then $n \geq 2$. Since the function $h_{2}(t)=(\ln t-\ln a)^{1-j}$ is decreasing and $\left(\frac{\ln t-\ln a}{\ln t}\right)^{\alpha-1} \leq 1$ for $\alpha>1$, we get, for $t \geq T_{2}$,

$$
\begin{aligned}
\left|(\ln t)^{1-\alpha} \Phi(t)\right| & =\left|(\ln t)^{1-\alpha} \Gamma(\alpha) \sum_{j=1}^{n} \frac{b_{j}\left(\ln \frac{t}{a}\right)^{\alpha-j}}{\Gamma(\alpha-j+1)}\right| \\
& \leq \Gamma(\alpha)\left(\frac{\ln t-\ln a}{\ln t}\right)^{\alpha-1} \sum_{j=1}^{n}\left|b_{j}\right| \frac{(\ln t-\ln a)^{1-j}}{\Gamma(\alpha-j+1)} \\
& \leq \Gamma(\alpha) \sum_{j=1}^{n}\left|b_{j}\right| \frac{(\ln t-\ln a)^{1-j}}{\Gamma(\alpha-j+1)} \\
& \leq \Gamma(\alpha) \sum_{j=1}^{n}\left|b_{j}\right| \frac{\left(\ln T_{2}-\ln a\right)^{1-j}}{\Gamma(\alpha-j+1)}:=c_{3}\left(T_{2}\right) .
\end{aligned}
$$

We also have

$$
\begin{aligned}
\left|(\ln t)^{1-\alpha} \Psi\left(t, T_{1}\right)\right| & =\left|(\ln t)^{1-\alpha} \int_{a}^{T_{1}}\left(\ln \frac{t}{s}\right)^{\alpha-1} \frac{\left[r(s)+f_{2}(s, x(s))-f_{1}(s, x(s))\right]}{s} d s\right| \\
& \leq \int_{a}^{T_{1}}\left(\frac{\ln t-\ln s}{\ln t}\right)^{\alpha-1} \frac{\left|r(s)+f_{2}(s, x(s))-f_{1}(s, x(s))\right|}{s} d s \\
& \leq \int_{a}^{T_{1}} \frac{\left|r(s)+f_{2}(s, x(s))-f_{1}(s, x(s))\right|}{s} d s \\
& :=c_{4}\left(T_{1}\right) .
\end{aligned}
$$

So, we conclude that

$$
(\ln t)^{1-\alpha} \int_{T_{1}}^{t}\left(\ln \frac{t}{s}\right)^{\alpha-1} \frac{r(s)}{s} d s \geq-\left[c_{3}\left(T_{2}\right)+c_{4}\left(T_{1}\right)\right]
$$


hence

$$
\liminf _{t \rightarrow \infty}(\ln t)^{1-\alpha} \int_{T_{1}}^{t}\left(\ln \frac{t}{s}\right)^{\alpha-1} \frac{r(s)}{s} d s \geq-\left[c_{3}\left(T_{2}\right)+c_{4}\left(T_{1}\right)\right]>-\infty
$$

which contradicts condition (15).

Therefore, we conclude that $x(t)$ is oscillatory. In case $x(t)$ is eventually negative, similar arguments lead to a contradiction with condition (16).

Theorem 3.3 Let conditions (10) and (11) hold with $\beta>\gamma$. If

$$
\liminf _{t \rightarrow \infty}(\ln t)^{1-\alpha} \int_{T}^{t}\left(\ln \frac{t}{s}\right)^{\alpha-1} \frac{[r(s)+H(s)]}{s} d s=-\infty
$$

and

$$
\limsup _{t \rightarrow \infty}(\ln t)^{1-\alpha} \int_{T}^{t}\left(\ln \frac{t}{s}\right)^{\alpha-1} \frac{[r(s)-H(s)]}{s} d s=\infty
$$

for every sufficiently large $T$, where

$$
H(s)=\frac{\beta-\gamma}{\gamma}\left[p_{1}(s)\right]^{\frac{\gamma}{\gamma-\beta}}\left[\frac{\gamma p_{2}(s)}{\beta}\right]^{\frac{\beta}{\beta-\gamma}},
$$

then every solution of $(1)$ is oscillatory.

Proof Let $x(t)$ be a nonoscillatory solution of equation (1), say, $x(t)>0$ for $t \geq T_{1}>a$. Let $s \geq T_{1}$. Using conditions (10) and (11), we get

$$
f_{2}(s, x)-f_{1}(s, x) \leq p_{2}(s) x^{\gamma}(s)-p_{1}(s) x^{\beta}(s) .
$$

Let $X=x^{\gamma}(s), Y=\frac{\gamma p_{2}(s)}{\beta p_{1}(s)}, u=\frac{\beta}{\gamma}$, and $v=\frac{\beta}{\beta-\gamma}$, then from part (i) of Lemma 2.1 we get

$$
\begin{aligned}
p_{2}(s) x^{\gamma}(s)-p_{1}(s) x^{\beta}(s) & =\frac{\beta p_{1}(s)}{\gamma}\left[x^{\gamma}(s) \frac{\gamma p_{2}(s)}{\beta p_{1}(s)}-\frac{\gamma}{\beta}\left(x^{\gamma}(s)\right)^{\frac{\beta}{\gamma}}\right] \\
& =\frac{\beta p_{1}(s)}{\gamma}\left[X Y-\frac{1}{u} X^{u}\right] \leq \frac{\beta p_{1}(s)}{\gamma} \frac{1}{v} Y^{v}=H(s),
\end{aligned}
$$

where $H$ is defined by (25). Then from equation (9) we obtain

$$
\begin{aligned}
\Gamma(\alpha) x(t) & =\Phi(t)+\Psi\left(t, T_{1}\right)+\int_{T_{1}}^{t}\left(\ln \frac{t}{s}\right)^{\alpha-1} \frac{\left[r(s)+f_{2}(s, x(s))-f_{1}(s, x(s))\right]}{s} d s \\
& \leq \Phi(t)+\Psi\left(t, T_{1}\right)+\int_{T_{1}}^{t}\left(\ln \frac{t}{s}\right)^{\alpha-1} \frac{\left[r(s)+p_{2}(s) x^{\gamma}(s)-p_{1}(s) x^{\beta}(s)\right]}{s} d s \\
& \leq \Phi(t)+\Psi\left(t, T_{1}\right)+\int_{T_{1}}^{t}\left(\ln \frac{t}{s}\right)^{\alpha-1} \frac{[r(s)+H(s)]}{s} d s .
\end{aligned}
$$

The rest of the proof is the same as that of Theorem 3.2 and hence is omitted. 
Theorem 3.4 Let $\alpha \geq 1$ and suppose that (10) and (12) hold with $\beta<\gamma$. If

$$
\limsup _{t \rightarrow \infty}(\ln t)^{1-\alpha} \int_{T}^{t}\left(\ln \frac{t}{s}\right)^{\alpha-1} \frac{[r(s)+H(s)]}{s} d s=\infty
$$

and

$$
\liminf _{t \rightarrow \infty}(\ln t)^{1-\alpha} \int_{T}^{t}\left(\ln \frac{t}{s}\right)^{\alpha-1} \frac{[r(s)-H(s)]}{s} d s=-\infty
$$

for every sufficiently large $T$, where $H$ is defined by (25), then every bounded solution of (1) is oscillatory.

Proof Let $x(t)$ be a bounded nonoscillatory solution of equation (1). Then there exist constants $M_{1}$ and $M_{2}$ such that

$$
M_{1} \leq x(t) \leq M_{2} \quad \text { for } t \geq a
$$

Assume that $x$ is a bounded eventually positive solution of (1). Then there exists $T_{1}>a$ such that $x(t)>0$ for $t \geq T_{1}>a$. Using conditions (10) and (12), we get $f_{2}(s, x)-f_{1}(s, x) \geq$ $p_{2}(s) x^{\gamma}(s)-p_{1}(s) x^{\beta}(s)$. Using (ii) of Lemma 2.1, and similar to the proof of (26), we find

$$
p_{2}(s) x^{\gamma}(s)-p_{1}(s) x^{\beta}(s) \geq H(s) \quad \text { for } s \geq T_{1} \text {. }
$$

From (9) and similar to (27), we obtain

$$
\Gamma(\alpha) x(t) \geq \Phi(t)+\Psi\left(t, T_{1}\right)+\int_{T_{1}}^{t}\left(\ln \frac{t}{s}\right)^{\alpha-1} \frac{[r(s)+H(s)]}{s} d s
$$

Multiplying by $(\ln t)^{1-\alpha}$, we get

$$
\begin{aligned}
(\ln t)^{1-\alpha} \Gamma(\alpha) x(t) \geq & (\ln t)^{1-\alpha} \Phi(t)+(\ln t)^{1-\alpha} \Psi\left(t, T_{1}\right) \\
& +(\ln t)^{1-\alpha} \int_{T_{1}}^{t}\left(\ln \frac{t}{s}\right)^{\alpha-1} \frac{[r(s)+H(s)]}{s} d s .
\end{aligned}
$$

Take $T_{2}>T_{1}$. We consider two cases as follows.

Case (1): Let $\alpha=1$. Then (19) and (20) are still correct. Hence, from (31) and using (30), we find that

$$
\begin{aligned}
M_{2} \Gamma(\alpha) \geq & (\ln t)^{1-\alpha} \Gamma(\alpha) x(t) \geq-c_{1}\left(T_{2}\right)-c_{2}\left(T_{1}, T_{2}\right) \\
& +(\ln t)^{1-\alpha} \int_{T_{1}}^{t}\left(\ln \frac{t}{s}\right)^{\alpha-1} \frac{[r(s)+H(s)]}{s} d s
\end{aligned}
$$

for $t \geq T_{2}$. Thus, we get

$$
\limsup _{t \rightarrow \infty}(\ln t)^{1-\alpha} \int_{T_{1}}^{t}\left(\ln \frac{t}{s}\right)^{\alpha-1} \frac{[r(s)+H(s)]}{s} d s \leq c_{1}\left(T_{2}\right)+c_{2}\left(T_{1}, T_{2}\right)+M_{2} \Gamma(\alpha)<\infty \text {, }
$$

which contradicts condition (28). 
Case (2): Let $\alpha>1$. Then (21) and (22) are still true. Hence, from (31) and using (30), we find that

$$
\begin{aligned}
M_{2} \Gamma(\alpha)(\ln t)^{1-\alpha} \geq & -c_{3}\left(T_{2}\right)-c_{4}\left(T_{1}\right) \\
& +(\ln t)^{1-\alpha} \int_{T_{1}}^{t}\left(\ln \frac{t}{s}\right)^{\alpha-1} \frac{[r(s)+H(s)]}{s} d s
\end{aligned}
$$

for $t \geq T_{2}$. Since $\lim _{t \rightarrow \infty}(\ln t)^{1-\alpha}=0$ for $\alpha>1$, we conclude that

$$
\limsup _{t \rightarrow \infty}(\ln t)^{1-\alpha} \int_{T_{1}}^{t}\left(\ln \frac{t}{s}\right)^{\alpha-1} \frac{[r(s)+H(s)]}{s} d s \leq c_{3}\left(T_{2}\right)+c_{4}\left(T_{1}\right)<\infty
$$

which contradicts condition (28). Therefore, we conclude that $x(t)$ is oscillatory. In case $x(t)$ is eventually bounded negative, similar arguments lead to a contradiction with condition (29).

\section{Oscillation of Hadamard fractional differential equations in the frame of \\ Caputo}

In this section, we study the oscillation of the Hadamard fractional differential equations in the Caputo setting of the form

$$
\left\{\begin{array}{l}
{ }^{C} \mathcal{D}_{a}^{\alpha} x(t)+f_{1}(t, x)=r(t)+f_{2}(t, x), \quad t>a \\
\delta^{k} x(a)=b_{k} \quad(k=0,1, \ldots, n-1),
\end{array}\right.
$$

where $n=\lceil\alpha\rceil$ and ${ }^{C} \mathcal{D}_{a}^{\alpha}$ is defined by (6).

Lemma 4.1 ([17]) Let $y \in A C_{\delta}^{n}[a, b]$ or $C_{\delta}^{n}[a, b]$ and $\alpha \in \mathbb{C}$. Then

$$
\left.\mathcal{I}_{a}^{\alpha}\left({ }^{C} \mathcal{D}_{a}^{\alpha}\right) y(x)\right)=y(x)-\sum_{k=0}^{n-1} \frac{\delta^{k} y(a)}{k !}\left(\ln \frac{x}{a}\right)^{k}
$$

Using Lemma 4.1, the solution representation of (32) can be written as

$$
x(t)=\sum_{k=0}^{n-1} \frac{\delta^{k} x(a)}{k !}\left(\ln \frac{t}{a}\right)^{k}+\mathcal{I}_{a}^{\alpha} F(t, x)
$$

where $F(t, x)=r(t)+f_{2}(t, x)-f_{1}(t, x)$.

Define

$$
\chi(t)=\Gamma(\alpha) \sum_{k=0}^{n-1} \frac{\delta^{k} x(a)}{k !}\left(\ln \frac{t}{a}\right)^{k}
$$

Theorem 4.2 Let $f_{2}=0$ in (32) and condition (10) hold. If

$$
\liminf _{t \rightarrow \infty}(\ln t)^{1-n} \int_{T}^{t}\left(\ln \frac{t}{s}\right)^{\alpha-1} \frac{r(s)}{s} d s=-\infty
$$


and

$$
\limsup _{t \rightarrow \infty}(\ln t)^{1-n} \int_{T}^{t}\left(\ln \frac{t}{s}\right)^{\alpha-1} \frac{r(s)}{s} d s=\infty
$$

for every sufficiently large $T$, then every solution of (32) is oscillatory.

Proof Let $x(t)$ be a nonoscillatory solution of equation (32) with $f_{2}=0$. Suppose that $T_{1}>a$ is large enough so that $x(t)>0$ for $t \geq T_{1}$. Hence (10) implies that $f_{1}(t, x)>0$ for $t \geq T_{1}$. Using (2), we get from (34)

$$
\begin{aligned}
\Gamma(\alpha) x(t)= & \Gamma(\alpha) \sum_{k=0}^{n-1} \frac{\delta^{k} x(a)}{k !}\left(\ln \frac{t}{a}\right)^{k} \\
& +\int_{a}^{T_{1}}\left(\ln \frac{t}{s}\right)^{\alpha-1} \frac{F(s, x(s))}{s} d s \\
& +\int_{T_{1}}^{t}\left(\ln \frac{t}{s}\right)^{\alpha-1} \frac{\left[r(s)-f_{1}(s, x(s))\right]}{s} d s \\
\leq & \Phi(t)+\Psi\left(t, T_{1}\right)+\int_{T_{1}}^{t}\left(\ln \frac{t}{s}\right)^{\alpha-1} \frac{r(s)}{s} d s
\end{aligned}
$$

where $\chi$ and $\Psi$ are defined in (35) and (14), respectively.

Multiplying (38) by $(\ln t)^{1-n}$, we get

$$
\begin{aligned}
0< & (\ln t)^{1-n} \Gamma(\alpha) x(t) \leq(\ln t)^{1-n} \chi(t)+(\ln t)^{1-n} \Psi\left(t, T_{1}\right) \\
& +(\ln t)^{1-n} \int_{T_{1}}^{t}\left(\ln \frac{t}{s}\right)^{\alpha-1} \frac{r(s)}{s} d s .
\end{aligned}
$$

Take $T_{2}>T_{1}$. We consider two cases as follows.

Case (1): Let $0<\alpha \leq 1$. Then $n=1$ and $(\ln t)^{1-n} \chi(t)=\Gamma(\alpha)$.

Since $h_{3}(t)=\left(\ln \frac{t}{s}\right)^{\alpha-1}$ is decreasing for $t>s$ and $t>T_{2}$, we get

$$
\begin{aligned}
\left|(\ln t)^{1-n} \Psi\left(t, T_{1}\right)\right| & =\left|\int_{a}^{T_{1}}\left(\ln \frac{t}{s}\right)^{\alpha-1} \frac{\left[r(s)+f_{2}(s, x(s))-f_{1}(s, x(s))\right]}{s} d s\right| \\
& \leq \int_{a}^{T_{1}}\left(\ln \frac{T_{2}}{s}\right)^{\alpha-1} \frac{\left|r(s)+f_{2}(s, x(s))-f_{1}(s, x(s))\right|}{s} d s \\
& :=c_{5}\left(T_{1}, T_{2}\right) .
\end{aligned}
$$

Then, from equation (39) and for $t \geq T_{2}$, we get

$$
(\ln t)^{1-n} \int_{T_{1}}^{t}\left(\ln \frac{t}{s}\right)^{\alpha-1} \frac{r(s)}{s} d s \geq-\left[\Gamma(\alpha)+c_{5}\left(T_{1}, T_{2}\right)\right]
$$

hence

$$
\liminf _{t \rightarrow \infty}(\ln t)^{1-n} \int_{T_{1}}^{t}\left(\ln \frac{t}{s}\right)^{\alpha-1} \frac{r(s)}{s} d s \geq-\left[\Gamma(\alpha)+c_{5}\left(T_{1}, T_{2}\right)\right]>-\infty,
$$

which contradicts condition (36). 
Case (2): Let $\alpha>1$. Then $n \geq 2$. Also, $\left(\frac{\ln t-\ln a}{\ln t}\right)^{n-1}<1$ for $n \geq 2$. The function $h_{4}(t)=$ $\left(\ln \frac{t}{a}\right)^{k-n+1}$ is decreasing for $k<n-1$. Thus, for $t \geq T_{2}$, we have

$$
\begin{aligned}
\left|(\ln t)^{1-n} \chi(t)\right| & =\left|(\ln t)^{1-n} \Gamma(\alpha) \sum_{k=0}^{n-1} \frac{\delta^{k} x(a)}{k !}\left(\ln \frac{t}{a}\right)^{k}\right| \\
& =\left|\left(\frac{\ln t-\ln a}{\ln t}\right)^{n-1} \Gamma(\alpha) \sum_{k=0}^{n-1} \frac{\delta^{k} x(a)}{k !}\left(\ln \frac{t}{a}\right)^{k-n+1}\right| \\
& \leq \Gamma(\alpha) \sum_{k=0}^{n-1} \frac{\left|\delta^{k} x(a)\right|}{k !}\left(\ln \frac{t}{a}\right)^{k-n+1} \\
& \leq \Gamma(\alpha) \sum_{k=0}^{n-1} \frac{\left|\delta^{k} x(a)\right|}{k !}\left(\ln \frac{T_{2}}{a}\right)^{k-n+1}:=c_{6}\left(T_{2}\right) .
\end{aligned}
$$

Also, since $\left(\frac{\ln t-\ln s}{\ln t}\right)^{n-1}<1$ for $n \geq 2$ and similar to (22), we get

$$
\left|(\ln t)^{1-\alpha} \Psi\left(t, T_{1}\right)\right| \leq c_{4}\left(T_{1}\right)
$$

Using the last inequality and (40), from (39) we get a contradiction with condition (36). Therefore, we conclude that $x(t)$ is oscillatory. In case $x(t)$ is eventually negative, similar arguments lead to a contradiction with condition (37).

We state the following two theorems without proof.

Theorem 4.3 Let conditions (10) and (11) hold with $\beta>\gamma$. If

$$
\liminf _{t \rightarrow \infty}(\ln t)^{1-n} \int_{T}^{t}\left(\ln \frac{t}{s}\right)^{\alpha-1} \frac{[r(s)+H(s)]}{s} d s=-\infty
$$

and

$$
\limsup _{t \rightarrow \infty}(\ln t)^{1-n} \int_{T}^{t}\left(\ln \frac{t}{s}\right)^{\alpha-1} \frac{[r(s)-H(s)]}{s} d s=\infty
$$

for every sufficiently large $T$, where $H$ is defined by (25), then every solution of (32) is oscillatory.

Theorem 4.4 Let $\alpha \geq 1$ and suppose that (10) and (12) hold with $\beta<\gamma$. If

$$
\limsup _{t \rightarrow \infty}(\ln t)^{1-n} \int_{T}^{t}\left(\ln \frac{t}{s}\right)^{\alpha-1} \frac{[r(s)+H(s)]}{s} d s=\infty
$$

and

$$
\liminf _{t \rightarrow \infty}(\ln t)^{1-n} \int_{T}^{t}\left(\ln \frac{t}{s}\right)^{\alpha-1} \frac{[r(s)-H(s)]}{s} d s=-\infty
$$

for every sufficiently large $T$, where $H$ is defined by (25), then every bounded solution of (32) is oscillatory. 


\section{Examples}

In this section, we construct examples to illustrate the effectiveness of our theoretical results.

Example 5.1 Consider the following Hadamard fractional differential equation in the Riemann setting:

$$
\left\{\begin{array}{l}
\mathcal{D}_{a}^{\alpha} x(t)+x^{5}(t) \ln (t+e) \\
\quad=\frac{2}{\Gamma(3-\alpha)}\left(\ln \frac{x}{a}\right)^{2-\alpha}+\left[\left(\ln \frac{x}{a}\right)^{10}-\left(\ln \frac{x}{a}\right)^{\frac{2}{3}}\right] \ln (t+e)+x^{\frac{1}{3}}(t) \ln (t+e) \\
\lim _{t \rightarrow a^{+}} \mathcal{D}_{a}^{\alpha-j} x(t)=0 \quad(j=1,2), 1<\alpha<2
\end{array}\right.
$$

where $n=2, f_{1}(t, x)=x^{5}(t) \ln (t+e), f_{2}(t, x)=x^{\frac{1}{3}}(t) \ln (t+e)$, and $r(t)=\frac{2}{\Gamma(3-\alpha)}\left(\ln \frac{x}{a}\right)^{2-\alpha}+$ $\left[\left(\ln \frac{x}{a}\right)^{10}-\left(\ln \frac{x}{a}\right)^{\frac{2}{3}}\right] \ln (t+e)$. It is easy to verify that conditions (10) and (11) are satisfied for $\beta=5, \gamma=\frac{1}{3}$, and $p_{1}(t)=p_{2}(t)=\ln (t+e)$. However, we show in the following that condition (23) does not hold. For every sufficiently large $T \geq 1$ and all $t \geq T$, we have $r(t)>0$. Calculating $H(s)$ as defined by (25), we find that $H(s)=14(15)^{-\frac{15}{14}} \ln (s+e) \geq 0.77$. Then, using (property 2.1) for $\beta=1$ and the fact that $\mathcal{D}^{\alpha}=\mathcal{I}^{-\alpha}$, we get

$$
\begin{aligned}
& \liminf _{t \rightarrow \infty}(\ln t)^{1-\alpha} \int_{T}^{t}\left(\ln \frac{t}{s}\right)^{\alpha-1} \frac{[r(s)+H(s)]}{s} d s \\
& \geq \liminf _{t \rightarrow \infty}(\ln t)^{1-\alpha} \int_{T}^{t}\left(\ln \frac{t}{s}\right)^{\alpha-1} \frac{H(s)}{s} d s \\
& \geq \liminf _{t \rightarrow \infty} 0.77(\ln t)^{1-\alpha} \int_{T}^{t}\left(\ln \frac{t}{s}\right)^{\alpha-1}\left(\ln \frac{s}{a}\right)^{0} \frac{d s}{s} \\
& =\liminf _{t \rightarrow \infty} 0.77(\ln t)^{1-\alpha} \Gamma(\alpha)\left(\mathcal{I}_{a}^{\alpha}\left(\ln \frac{s}{a}\right)^{0}\right)(t) \\
& =\liminf _{t \rightarrow \infty} 0.77 \frac{\ln t}{\alpha}\left(1-\frac{\ln a}{\ln t}\right)^{\alpha}=\infty .
\end{aligned}
$$

However, one can easily verify that $x(t)=\left(\ln \frac{t}{a}\right)^{2}$ is a nonoscillatory solution of (45). The initial condition is also satisfied because

$$
\mathcal{D}_{a}^{\alpha-j}\left(\ln \frac{t}{a}\right)^{2}=\mathcal{I}_{a}^{j-\alpha}\left(\ln \frac{t}{a}\right)^{2}=\frac{2}{\Gamma(3+j-\alpha)}\left(\ln \frac{t}{a}\right)^{2+j-\alpha}, \quad j=1,2 .
$$

Example 5.2 Consider the Hadamard fractional differential equation

$$
\left\{\begin{array}{l}
\mathcal{D}_{a}^{\alpha} x(t)+x^{3}(t)=\sin t, \\
\lim _{t \rightarrow a^{+}} \mathcal{D}_{a}^{\alpha-1} x(t)=0, \quad 0<\alpha<1,
\end{array}\right.
$$

where $f_{1}(t, x)=x^{3}(t), r(t)=\sin t$, and $f_{2}(t, x)=0$. Then condition (10) holds. Furthermore, one can easily check that

$$
\liminf _{t \rightarrow \infty}(\ln t)^{1-\alpha} \int_{T}^{t}\left(\ln \frac{t}{s}\right)^{\alpha-1} \frac{\sin s}{s} d s=-\infty
$$


and

$$
\limsup _{t \rightarrow \infty}(\ln t)^{1-\alpha} \int_{T}^{t}\left(\ln \frac{t}{s}\right)^{\alpha-1} \frac{\sin s}{s} d s=\infty
$$

This shows that conditions (15) and (16) of Theorem 3.2 hold. Hence, every solution of (46) is oscillatory.

Example 5.3 Consider the following Hadamard fractional differential equation of Caputo type:

$$
\left\{\begin{array}{l}
{ }^{C} \mathcal{D}_{a}^{\alpha} x(t)+e^{t} x^{3}(t)=\frac{1}{\Gamma(2-\alpha)}\left(\ln \frac{t}{a}\right)^{1-\alpha}+\left(\ln \frac{t}{a}\right)^{3} e^{t} \\
x(a)=0, \quad \delta x(a)=1, \quad 1<\alpha<2
\end{array}\right.
$$

where $n=2, f_{1}(t, x)=e^{t} x^{3}(t), r(t)=\frac{1}{\Gamma(2-\alpha)}\left(\ln \frac{t}{a}\right)^{1-\alpha}+\left(\ln \frac{t}{a}\right)^{3} e^{t}$, and $f_{2}(t, x)=0$. Then condition (10) is satisfied. Since $r(s)>0$ and using (2), we get

$$
\begin{aligned}
& \liminf _{t \rightarrow \infty}(\ln t)^{1-n} \int_{T}^{t}\left(\ln \frac{t}{s}\right)^{\alpha-1} \frac{r(s)}{s} d s \\
& \geq \liminf _{t \rightarrow \infty}(\ln t)^{1-n} \int_{T}^{t}\left(\ln \frac{t}{s}\right)^{\alpha-1}\left(\ln \frac{s}{a}\right)^{0} \frac{d s}{s} \\
& =\liminf _{t \rightarrow \infty}(\ln t)^{1-m} \Gamma(\alpha)\left(\mathcal{I}_{a}^{\alpha}\left(\ln \frac{s}{a}\right)^{0}\right)(t) \\
& =\liminf _{t \rightarrow \infty}(\ln t)^{1-n} \frac{\Gamma(\alpha)}{\Gamma(1+\alpha)}\left(\ln \frac{t}{a}\right)^{\alpha} \\
& =\liminf _{t \rightarrow \infty} \frac{\Gamma(\alpha)}{\Gamma(1+\alpha)}\left(\ln \frac{t}{a}\right)^{\alpha-n+1}\left(1-\frac{\ln a}{\ln t}\right)^{n-1} \\
& =\infty,
\end{aligned}
$$

which means that condition (36) does not hold. However, it is forward to check that $x(t)=$ $\ln \frac{t}{a}$ is a nonoscillatory solution of (47).

\section{Conclusion}

In this article, the oscillation theory for Hadamard fractional differential equations was studied. Sufficient conditions for the oscillation of solutions of Hadamard fractional differential equations in the Riemann setting (1) were given in three theorems in Sect. 3. The main approach is based on applying Young's inequality which will help us in obtaining sharper conditions. The oscillation for the Hadamard fractional differential equations in the Caputo setting has been investigated as well. Numerical examples are presented to demonstrate the effectiveness of the obtained results. We finally remark that dual oscillation properties for fractional systems in the frame of right Hadamard fractional derivatives given in (5) can be concluded from the left cases through applying the Q-operator. It would be of interest, in future works, to study the oscillation of other types of fractional systems in the frame of fractional derivatives with more generalized kernels or nonsingular kernels [24]. 


\section{Funding}

The authors would like to thank Prince Sultan University for funding this work through research group Nonlinear Analysis Methods in Applied Mathematics (NAMAM), group number RG-DES-2017-01-17.

\section{Competing interests}

The authors declare that they have no competing interests.

\section{Authors' contributions}

All authors contributed equally and significantly to this paper. All authors have read and approved the final manuscript.

\section{Publisher's Note}

Springer Nature remains neutral with regard to jurisdictional claims in published maps and institutional affiliations.

\section{Received: 24 July 2018 Accepted: 31 October 2018 Published online: 07 November 2018}

\section{References}

1. Alzabut, J., Abdeljawad, T., Alrabaiah, H.: Oscillation criteria for forced and damped nabla fractional difference equations. J. Comput. Anal. Appl. 24(8), 1387-1394 (2018)

2. Grace, S.R., Agarwal, R.P., Wong, P.J.Y., Zafer, A.: On the oscillation of fractional differential equations. Fract. Calc. Appl. Anal. 15, 222-231 (2012). https://doi.org/10.2478/s13540-012-0016-1

3. Chen, D.-X., Qu, P.-X., Lan, Y.-H.: Forced oscillation of certain fractional differential equations. Adv. Differ. Equ. 2013, 125 (2013). https://doi.org/10.1186/1687-1847-2013-125

4. Alzabut, J., Abdeljawad, T.: Sufficient conditions for the oscillation of nonlinear fractional difference equations. J. Fract. Calc. Appl. 5(1), 177-178 (2014)

5. Abdalla, B., Abodayeh, K., Abdeljawad, T., Alzabut, J.: New oscillation criteria for forced nonlinear fractional difference equations. Vietnam J. Math. 45(4), 609-618 (2017). https://doi.org/10.1007/s10013-016-0230-y

6. Abdalla, B.: On the oscillation of q-fractional difference equations. Adv. Differ. Equ. 2017, 254 (2017) https://doi.org/10.1186/s13662-017-1316-x

7. Abdalla, B.: Oscillation of differential equations in the frame of nonlocal fractional derivatives generated by conformable derivatives. Adv. Differ. Equ. 2018, 107 (2018). https://doi.org/10.1186/s13662-018-1554-6

8. Abdalla, B., Alzabut, J., Abdeljawad, T.: On the oscillation of higher order fractional difference equations with mixed nonlinearities. Hacet. J. Math. Stat. 47(2), 207-217 (2018). https://doi.org/10.15672/HJMS.2017.458

9. Khan, H., Tunç, C., Chen, W., Khan, A.: Existence theorems and Hyers-Ulam stability for a class of Hyberd fractional differential equations with P-Laplacian operator. J. Appl. Anal. Comput. 8(4), 1211-1226 (2018)

10. Khan, A., Syam, M.I., Zada, A., Khan, H.: Stability analysis of nonlinear fractional differential equations with Caputo and Riemann-Liouville derivatives. Eur. Phys. J. Plus 133, 264 (2018). https://doi.org/10.1140/epjp/i2018-12119-6

11. Khan, H., Chen, W., Sun, H.: Analysis of positive solution and Hyers-Ulam stability for a class of singular fractional differential equations with p-Laplacian in Banach space, Math. Methods Appl. Sci. 41, 3430-3440 (2018). https://doi.org/10.1002/mma.4835

12. Akgul, A., Sajjad Hashemi, M., Inc, M., Baleanu, D., Khan, H.: New method for investigating the density-dependent diffusion Nagumo equation. Therm. Sci. 22(Suppl. 1), S143-S152 (2018)

13. Baleanu, D., Agarwal, R.P., Khan, H., Khan, R.A., Jafari, H.: On the existence of solution for fractional differential equations of order $3<\delta_{1}<4$. Adv. Differ. Equ. 2015, Article ID 362 (2015).

14. Baleanu, D., Golmankhaneh, A.K., Golmankhaneh, A.K., Nigmatullin, R.R.: Newtonian law with memory. Nonlinear Dyn. 60(1-2), 81-86 (2009)

15. Golmankhaneh, A.K., Golmankhaneh, A.K., Baleanu, D.: On nonlinear fractional Klein-Gordon equation. Signal Process. 91(3), 446-451 (2011)

16. Hadamarad, J.: Essai sur l'etude des fonctions donnes par leur developpment de Taylor. J. Pure Apple Math. 4(8), $101-186(1892)$

17. Jarad, F., Abdeljawad, T., Baleanu, D.: Caputo-type modification of the Hadamard fractional derivatives. Adv. Differ Equ. 2012, $142(2012)$

18. Adjabi, Y., Jarad, J.F., Baleanu, D., Abdeljawad, T.: On Cauchy problems with Caputo Hadamard fractional derivatives J. Comput. Anal. Appl. 21(1), 661-681 (2016)

19. Gambo, Y.Y., Jarad, F., Baleanu, D., Abdeljawad, T:: On Caputo modification of the Hadamard fractional derivatives. Adv. Differ. Equ. 2014, 10 (2014)

20. Kilbas, A.A., Srivastava, M.H., Trujillo, J.J.: Theory and Application of Fractional Differential Equations. North Holland Mathematics Studies, vol. 204 (2006)

21. Abdeljawad, T., Jarad, F., Baleanu, D.: On the existence and the uniqueness theorem for fractional differentia equations with bounded delay within Caputo derivatives. Sci. China Ser. A, Math. 51(10), 1775-1786 (2008)

22. Abdeljawad, T., Baleanu, D., Jarad, F.: Existence and uniqueness theorem for a class of delay differential equations with left and right Caputo fractional derivatives. J. Math. Phys.. 49(8), 083507 (2008)

23. Hardy, G.H., Littlewood, J.E., Polya, G.: Inequalities, 2nd edn. Cambridge University Press, Cambridge (1988)

24. Abdeljawad, T., Baleanu, D.: Integration by parts and its applications of a new nonlocal fractional derivative with Mittag-Leffler nonsingular kernel. J. Nonlinear Sci. Appl. 10(3), 1098-1107 (2017) 